University of South Carolina

Scholar Commons

1994

\title{
Effect of Proton Diffusion, Electron Conductivity, and Charge- Transfer Resistance on Nickel Hydroxide Discharge Curves
}

John W. Weidner

University of South Carolina - Columbia, weidner@engr.sc.edu

Paul Timmerman

California Institute of Technology

Follow this and additional works at: https://scholarcommons.sc.edu/eche_facpub

Part of the Chemical Engineering Commons

\section{Publication Info}

Journal of the Electrochemical Society, 1994, pages 346-351.

(c) The Electrochemical Society, Inc. 1994. All rights reserved. Except as provided under U.S. copyright law, this work may not be reproduced, resold, distributed, or modified without the express permission of The Electrochemical Society (ECS). The archival version of this work was published in the Journal of the Electrochemical Society.

http://www.electrochem.org/

Publisher's link: http://dx.doi.org/10.1149/1.2054729

DOI: $10.1149 / 1.2054729$

This Article is brought to you by the Chemical Engineering, Department of at Scholar Commons. It has been accepted for inclusion in Faculty Publications by an authorized administrator of Scholar Commons. For more information, please contact digres@mailbox.sc.edu. 


\section{REFERENCES}

1. A. O. Isenberg, in Proceedings of International Symposium Fine Ceramics, Arita, Vol. 88, p. 105 (1988).

2. I. Kudo and H. Obayashi, This Journal, 123, 415 (1976).

3. T. Takahashi and H. Iwahara, Energy Conver, 11, 359 (1971).

4. H. Yahiro, Y. Eguchi, K. Eguchi, and H. Arai, J. Appl. Electrochem., 18, 527 (1988).

5. I. Anzai, N. Sakai, T. Kawada, H. Yokokawa, and M. Dokiya, in Proceedings of 31 st Battery Symposium, Jpn., 2 C04 (1990).

6. T. Takahashi, H. Iwahara, and I. Ito, Denki Kagaku, 38, 509 (1970).
7. O. Yamamoto, Y. Takeda, R. Kanno, and M. Noda, Solid State Ionics, 22, 241 (1987)

8. Y. Takeda, R. Kanno, M. Noda, Y. Tomida, and O. Yamamoto, This Journal, 134, 2656 (1987).

9. O. Yamamoto, Y. Takeda, R. Kanno, and Y. Tomida, Nippon Kagaku Kaishi, 1324 (1988).

10. M. Watanabe, T. Saegusa, and P. Stonehart, Chem. Lett., 1467 (1988); J. Electroanal. Chem., 271, 213 (1989); M. Watanabe, H. Sei, and P. Stonehart, ibid., 261, 375 (1989).

11. A. Hamouche, E. Siebert, A. Hammou, M. Kleitz, and A. Caneiro, This Journal, 138, 1212 (1991).

12. M. Liu and A. Khandkar, Solid State Ionics, 52, 3 (1992).

\title{
Effect of Proton Diffusion, Electron Conductivity, and Charge-Transfer Resistance on Nickel Hydroxide Discharge Curves
}

\author{
John W. Weidner* \\ Department of Chemical Engineering, University of South Carolina, Columbia, South Carolina 29208 \\ Paul Timmerman* \\ Jet Propulsion Laboratory, California Institute of Technology, Pasadena, California 91109
}

\section{ABSTRACT}

Constant-current discharge curves for the nickel hydroxide electrode are simulated assuming resistances due to diffusion of protons and conduction of electrons through the nickel hydroxide film, and charge-transfer resistance at the film/electrolyte interface contribute to the polarization losses of the electrode. Good qualitative agreement is observed between the model predictions and experimental discharge curves. The results suggest that polarization losses due to diffusional limitations of protons is a critical factor in determining the characteristics of the discharge curve. Ohmic resistance has a significant effect on the discharge curves at the end of discharge, and charge-transfer resistance is a minor contributor to the polarization losses. These findings indicate that accurately measuring the diffusion coefficient of protons, the thickness of the hydroxide film, the initial state-of-charge, and the electronic conductivity as a function of state-of-charge towards the end of discharge are critical in accurately predicting the discharge characteristics of nickel hydroxide. Physical constants which were shown to have minor influence on the discharge curves are the film conductivity at the beginning of discharge, and the exchange current density and cathodic transfer coefficient for the reaction. The time-dependent, one-dimensional diffusion equation has been solved analytically which should provide a computationally efficient means of accounting for proton diffusion and variable electronic conductivity in a macrohomogeneous battery model without sacrificing accuracy.

Battery models that can predict the effect of operating conditions on battery life and performance are extremely valuable to battery users and manufacturers. Model predictions can provide quality assurance that the batteries being manufactured are of consistently high quality, and provide indicators well in advance of failure so that steps can be taken to adjust operating condition and prolong battery life. In addition, a variety of design parameters can be investigated to aid an electrode development program.

Theoretical discharge curves for nickel hydroxide have been generated by a number of investigators ${ }^{1-5}$ using a onedimensional, macrohomogeneous model. The spatial dimension of interest is in the direction perpendicular to the current collectors, and therefore the polarization losses caused by the $\mathrm{NiOOH} / \mathrm{Ni}(\mathrm{OH})_{2}$ film is assumed to be due solely to a pseudocharge-transfer resistance. Although these models reveal the importance of transport limitations in the electrolyte phase, they predict nickel electrode potentials as a function of time which are more positive and flatter than experimental data. They also predict material utilization on discharge which is unrealistically high. Sinha ${ }^{6}$ developed a model of a porous electrode in which the solid material was described using semiconductor the-

* Electrochemical Society Active Member. ory. This model, however, contains many parameters that cannot be obtained experimentally.

Experimentally it has been suggested that resistance due to diffusion of protons and/or conduction of electrons may contribute appreciably to overall polarization losses at the nickel hydroxide film. ${ }^{710}$ Two-dimensional, macrohomogeneous models have been developed to account for the diffusion of protons in the active nickel hydroxide film ${ }^{11}$ and diffusion coupled with variable electronic resistance of the film. ${ }^{12}$ One of the rationales for including variable electronic resistance in the later model is that nickel hydroxide is an electrical insulator in the reduced state and a conductor in the oxidized state. The drawback of these models is that the solution procedure requires a large amount of computer memory and computational time.

In this paper, an analytical solution to the time-dependent, one-dimensional diffusion equation is incorporated into a discharge model which accounts for proton diffusion and variable electronic conductivity in the film, and charge-transfer resistance at the film/electrolyte interface. The governing equations are presented in such a manner that they can be used in conjunction with a one-dimensional, macrohomogeneous model in order to predict the behavior of an entire nickel battery. The results presented here though ignore: $(i)$ the interaction among the positive 


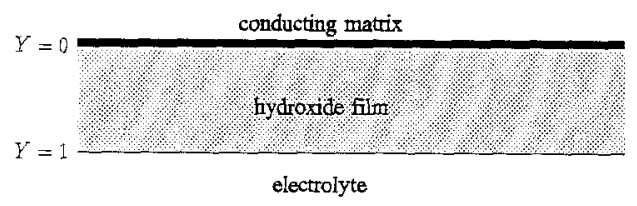

Fig. 1. A schematic of the active nickel hydroxide film. The film is assumed to deposit uniformly on a conducting porous matrix with a thickness $\mathrm{I}$. The $Y$ coordinate is normalized with respect to $I$. The electrolyte is assumed to be $31 \% \mathrm{KOH}$.

and negative electrodes, and the separator, (ii) a nonuniform reaction distribution, and (iii) side reactions such as those involving oxygen and hydrogen. The reason for these omissions is not to argue that their contribution is unimportant, but rather the objective of this paper is to isolate the effect of proton diffusion, electron conductivity, and charge-transfer resistance on nickel hydroxide discharge curves. Charge curves are not presented since critical qualitative trends cannot be observed without modeling the entire cell. For example, continuous overcharge is possible for a nickel/cadmium battery due to oxygen evolution at the positive (nickel) electrode, transport of oxygen across the separator, and reduction of oxygen at the negative (cadmium) electrode. ${ }^{4,5}$

\section{Mathematical Model}

Figure 1 shows a schematic of the active nickel hydroxide film. The active material is assumed to be uniformly impregnated on a conducting porous matrix. The discharge reaction at the film/electrolyte interface $(Y=1)$ is generally expressed as

$$
\mathrm{NiOOH}+\mathrm{H}_{2} \mathrm{O}+e^{-} \rightarrow \mathrm{Ni}(\mathrm{OH})_{2}+\mathrm{OH}^{-}
$$

Proton diffusion into the bulk of the solid phase makes it possible for this reaction to continue at the interface. $\mathrm{NiOOH}$ and $\mathrm{Ni}(\mathrm{OH})_{2}$ are assumed to coexist in the hydroxide film in a single phase rather than two separate phases. The relative activities in the solid phase are approximated by their respective mole fractions, $x_{j}$, and species other than $\mathrm{NiOOH}$ and $\mathrm{Ni}(\mathrm{OH})_{2}$ are negligible. It is further assumed that the local degree of discharge is equal to the mole fraction of the $\mathrm{Ni}(\mathrm{OH})_{2}$ species and related to the dimensionless proton concentration, $C$, as follows

$$
x_{\mathrm{Ni}(\mathrm{OH})_{2}}=1-x_{\mathrm{NiOOH}}=C\left(1-\theta^{\circ}\right)
$$

where $\theta^{\circ}$ is the initial state-of-charge. (See the List of Symbols and Table I for a complete list of variables and parameter definitions.)

The above assumptions have been used by previous investigators $\mathrm{s}^{5,12}$ to yield a kinetic expression which relates the current to the potential driving force and proton concentration at the film/electrolyte interface. The expression can be written in dimensionless form as

$$
I(\tau)=I_{0}\left\{C(1, \tau)\left(1-\theta^{\circ}\right) e^{(1-\alpha) f n}-\left[1-C(1, \tau)\left(1-\theta^{\circ}\right)\right] e^{-\alpha f \eta}\right\}
$$

where $\eta$ is the overpotential at the film/electrolyte interface relative to a reference potential and defined as

\section{Table I. The definitions and physical interpretations of the

\begin{tabular}{|c|c|c|}
\hline Parameter & Interpretation & Definition \\
\hline$I_{\mathrm{o}}$ & $\frac{\text { Discharge time }}{\text { Charge-transfer resistance }}$ & $\frac{3600 n^{\prime} i_{0, \text { ref }}}{F C_{\mathrm{Ni}} l}$ \\
\hline$\alpha$ & Cathodic transfer coefficient & \\
\hline $\mathscr{D}$ & $\frac{\text { Discharge time }}{\text { Diffusion resistance }}$ & $\frac{3600 n^{\prime} D_{\mathrm{H}^{+}}}{l^{2}}$ \\
\hline$\theta^{\circ}$ & Initial state-of-charge & \\
\hline$\sigma^{*}$ & $\frac{\text { Discharge time }}{\text { Ohmic resistance }}$ & $\frac{3600 n^{\prime} \sigma^{\circ}}{f F C_{\mathrm{N} l} l^{2}}$ \\
\hline
\end{tabular} dimensionless parameters that govern nicke hydroxide discharge curves.}

$$
\eta(\tau) \equiv\left(\phi_{\mathrm{s}}-\phi_{1}-U_{\mathrm{ref}}\right)
$$

$\left(U_{\text {ref }}=0.39 \mathrm{~V} v s\right.$. HgO-Hg, the equilibrium potential for Eq. 1 at $\theta=0.5$ and $T=293 \mathrm{~K}$ in $31 \% \mathrm{KOH}){ }^{13}$

In order to obtain the potential in the matrix phase, Ohm's law is applied in the hydroxide layer. Integrating from the conducting matrix $(Y=0)$ to the electrolyte $(Y=1)$ yields

$$
\begin{aligned}
\left(\phi_{\mathrm{m}}-\phi_{1}\right)=\left(\phi_{\mathrm{s}}-\phi_{1}\right)+\frac{1}{f} & {\left[\frac{I(\tau)}{\sigma^{*}} \int_{0}^{1}\right.} \\
& \left.\quad \exp \left[24.45 C(Y, \tau)^{4}\left(1-\theta^{\circ}\right)^{4}\right] d Y\right]
\end{aligned}
$$

In general, the total current (e.g., nickel and oxygen reduction) would be included in Eq. 5. In this paper, nickel reduction (Eq. 1) is assumed to be the only reaction.

The integral expression in Eq. 5 arises from the assumption that the conductivity of the hydroxide film varies locally depending on the local state-of-charge. ${ }^{12}$ The form of the equation is empirical and reflects the fact that pure $\mathrm{Ni}(\mathrm{OH})_{2}$ is an insulator and the oxidized form is a conductor. Other expressions can be used to obtain subtle features in the predicted discharge curves. For example, Mao et al. ${ }^{12}$ added a discontinuity in the conductivity which gave rise to a secondary discharge plateau which is observed experimentally. ${ }^{14}$ The qualitative trends presented in this paper do not depend on the form of the integral in Eq. 5 and, therefore, results using different conductivity expressions are not given.

In order to generate a discharge curve using Eq. 3 and 5, it is necessary to know the proton concentration profile at every point in time. If it is assumed that protons incorporated into the hydroxide film due to Eq. 1 will move by diffusion and that the diffusion coefficient is not a function of state-of-charge, a concentration profile can be obtained by solving the time-dependent, one-dimensional diffusion equation. In dimensionless form this equation can be written as

$$
\frac{\partial C}{\partial \tau}=\frac{\partial^{2} C}{\partial Y^{2}}
$$

The initial proton concentration is assumed to be uniform throughout the film, the proton concentration gradient is zero at the interface between the conducting matrix and the active material $(Y=0)$, and the concentration gradient is proportional to the current at the film/electrolyte interface $(Y=1)$. The initial and boundary conditions can be written as

$$
\begin{gathered}
\tau=0 \quad C=1 \\
Y=0 \quad \frac{\partial C}{\partial Y}=0 \\
Y=1 \quad \frac{\partial C}{\partial Y}=\frac{-I(\tau)}{\mathscr{D}\left(1-\theta^{\circ}\right)}
\end{gathered}
$$

where $I(\tau)$ is the dimensionless current density which is negative for a cathodic (discharge) current.

The Laplace transform of Eq. 6 is taken with respect to $\tau$, the resulting ordinary differential equation for the transformed concentration is solved, and the convolution property of Laplace transforms is applied to invert back to the time domain to yield

$$
\begin{aligned}
C(Y, \tau)=1- & \frac{1}{\mathscr{D}\left(1-\theta^{\circ}\right)} \int_{0}^{\tau} \\
& {\left[1+2 \sum_{k=1}^{\infty}(-1)^{k} e^{-k^{2} \pi^{2}(\tau-z)} \cos (k \pi Y)\right] I(z) d z }
\end{aligned}
$$

Known current density.--If the discharge current is constant, and the reaction is uniform throughout the porous matrix, $I(z)=-1$ and Eq. 7 can be integrated to give

$$
C(Y, \tau)=1+\frac{1}{\mathscr{g}\left(1-\theta^{\circ}\right)}
$$

$$
\left[\tau+2 \sum_{k=1}^{\infty} \frac{(-1)^{k} \cos (k \pi Y)}{k^{2} \pi^{2}}\left(1-e^{-k^{2} \pi^{2} \tau}\right)\right]
$$




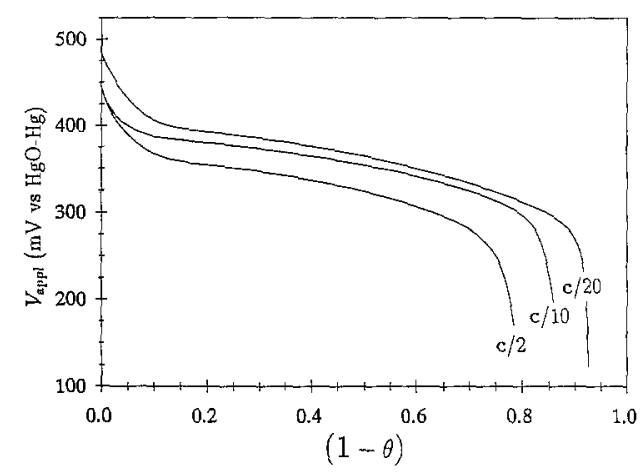

Fig. 2. The effect of discharge rate on experimental nickel hydroxide discharge curves obtained from nickel/cadmium cells $(T=293)$. The term $c / n$ is the current which causes the nominal capacity of the electrode to completely discharge in $n$ hours. As $n$ increases, the discharge rate decreases. The applied voltages are plotted as a function of the averoge degree-of-discharge.

In addition, $\left(\phi_{\mathrm{m}}-\phi_{1}\right)$ will be equal to the applied voltage, $V_{\text {appl. }}$.

Unknown current density.-If $I(z)$ is not known, Eq. 7 cannot be integrated directly. Examples of how an unknown $I(z)$ could arise are a potential-controlled discharge, or a current-controlled discharge where the reaction is nonuniform. The latter case would apply when incorporating the effect of proton diffusion into a macrohomogeneous battery model. Although the overall current is dictated by the discharge rate, the local current density is affected by local potential and hydroxide-ion concentration variations. For an unknown current density, the integral in Eq. 7 can be approximated numerically ${ }^{15}$ as

$$
\begin{gathered}
\int_{0}^{\tau}\left[1+2 \sum_{k=1}^{\infty}(-1)^{k} e^{-k^{2} \pi^{2}(\tau-z)} \cos (k \pi Y)\right] \\
I(z) d z \approx \sum_{\mu=0}^{q} B_{\mu}(Y) I(q h-\mu h)
\end{gathered}
$$

where $h$ is the interval width such that $\tau=q h$ and $z=\mu h$. The coefficients $B_{\mu}$ are determined by approximating the unknown function $I(z)$ by a linear expression in the interval between $\mu$ and $\mu-1$, integrating the left side of Eq. 9, and solving for the coefficients to yield

$$
\begin{aligned}
& B_{0}(Y)=\frac{h}{2}-2 \sum_{k=1}^{\infty} \frac{(-1)^{k} \cos (k \pi Y)}{k^{4} \frac{\pi^{4} h}{4}}\left(1-e^{-k^{2} \pi^{2} h}-k^{2} \pi^{2} h\right) \\
& B_{\mu}(Y)=h+2 \sum_{k=1}^{\infty} \frac{(-1)^{\grave{k}} \cos (k \pi Y) e^{-k^{2} \pi^{2} h(\mu-1)}}{k^{4} \pi^{4} h}\left(1-e^{-k^{2} \pi^{2} h}\right)^{2}
\end{aligned}
$$

for $\mu$ from 1 to $q-1$, and

$$
\begin{aligned}
& B_{\mathrm{q}}(Y)=\frac{h}{2}-2 \sum_{k=1}^{\infty} \frac{(-1)^{k} \cos (k \pi Y) e^{-k^{2} \pi^{2} h(\mathrm{q}-1)}}{k^{4} \pi^{4} h} \\
& {\left[e^{-k^{2} \pi^{2} h}\left(1+k^{2} \pi^{2} h\right)-1\right]}
\end{aligned}
$$

for $q \neq 0$. Substituting Eq. 9 into Eq. 7 yields

$$
\begin{aligned}
C(Y, \tau)=1-\frac{1}{\mathscr{D}\left(1-\theta_{0}\right)} & \\
& {\left[B_{0}(Y) I(\tau)+\sum_{\mu=1}^{\mathrm{q}} B_{\mu}(Y) I(q h-\mu h)\right] }
\end{aligned}
$$

\section{Results and Discussion}

Experimental results.-Figure 2 shows experimental nickel hydroxide discharge curves obtained from nickel/ cadmium cells. The term $\mathbf{c} / n$ is the current which causes the nominal capacity of the cell to discharge in $n$ hours. The details of the test method used to obtain these curves have been described previously. ${ }^{16}$ These cells, consisting of 11 negative and 12 positive plates, had a nominal capacity of

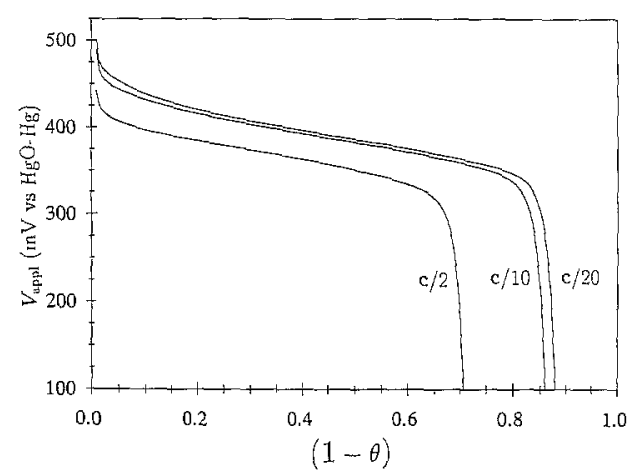

Fig. 3. The effect of discharge rate on simulated nickel hydroxide discharge curves. The curves were generated by solving Eq. 3, 5, and 8. The term $c / n$ is the current which causes the nominal capacity of the electrode to completely discharge in $n$ hours. As $n$ increases, the discharge rate decreases. The applied voltages are plotted as a function of the average degree-of-discharge. The physical consfants used to generate these curves are shown in Table II resulting in: $\sigma^{*}=$ $15,000 n ; I_{0}=1.5 n ; \mathscr{D}=0.87 n$.

26.5 Ah. Therefore, a c/2 discharge rate corresponds to a discharge current of $13.25 \mathrm{~A}$ which is approximately 11 $\mathrm{mA} / \mathrm{cm}^{2}$. The dimensionless parameters listed in Table I and the dimensionless current, however, are defined relative to the hours required to completely discharge the actual capacity. The experimental cells had an actual capacity of 34.87 Ah which means a $c / 2$ rate would take $2.63 \mathrm{~h}$ to completely discharge these cells. The ratio of the nominal to actual capacity gives a $n / n$ ' ratio of 0.76 which is used in simulating the discharge curves in order to make the experimental rates compatible with those used in the model. In plotting the discharge curves in Fig. 2, the electrodes are assumed to start fully charged, and $1-\theta$ is determined by dividing the product of current and time by the actual capacity.

Three characteristics of the discharge curve are seen in Fig. 2: (i) a quickly decaying voltage during the early portion of discharge; (ii) a voltage plateau during the middle portion of discharge which decreases as the discharge rate increases; (iii) material utilization which decreases as discharge rate increases. Similar trends have been reported previously ${ }^{16}$ but there are quantitative differences between those results and the ones shown in Fig. 2. Cycling hysteresis, for example, is a critical factor in determining the potential of the electrode at a given degree-of-discharge. It is also not known if the electrode is fully recharged after discharge. It will be shown later when discussing the effect $\theta^{\circ}$ has on the discharge curves that accurately determine this physical constant prior to discharge is critical if quantitative comparisons between experimental and simulated curves are to be made. In addition, thermal affects can influence the discharge curves, especially at high rates. Therefore, the three characteristics described above are used to make qualitative comparisons between the experimental and simulated discharge curves. These comparisons provide insight into the relative contribution of masstransfer, ohmic, and charge-transfer resistance to the polarization losses.

Known current density.-Figure 3 shows the effect of discharge rate on simulated nickel hydroxide discharge

Table II. The physical consiants used to obtain the dimensionless parameters given in Fig. 3 and the curves corresponding to these parameters.

$\begin{array}{ll}c_{\mathrm{Ni}}=0.040 \mathrm{~mol} / \mathrm{cm}^{3} & D_{\mathrm{H}^{+}}=4.6 \times 10^{-11} \mathrm{~cm}^{2} / \mathrm{s} \\ i_{\mathrm{o} \text {,ef }}=6.0 \times 10^{-4} \mathrm{~A} / \mathrm{cm}^{2} & l=5.0 \times 10^{-4} \mathrm{~cm} \\ \sigma^{\circ}=0.1185(\Omega \cdot \mathrm{cm})^{-1} & T=293 \mathrm{~K} \\ \alpha=0.5 & \theta^{\circ}=0.99 \\ n / n^{\prime}=0.76 & \end{array}$




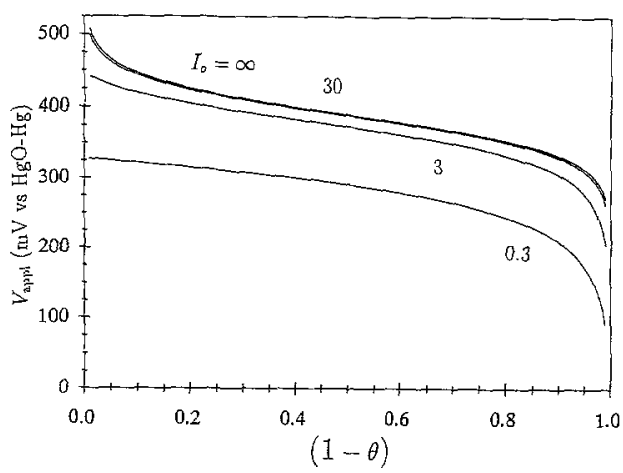

Fig. 4. The effect of charge-transfer resistance on nickel hydroxide discharge curves. As $l_{0}$ increases, charge-transfer resistance decreases relative to discharge time. The applied voltages are plotted as a function of the average degree-of-discharge. $\sigma^{*}=\mathscr{D}=\infty ; \alpha=0.5$; $\theta^{\circ}=0.99 ; T=293$.

curves. Each curve was obtained by calculating $C(1, \tau)$ using Eq. 8, solving Eq. 3 for $\eta(\tau)$, obtaining the applied voltage $\left(V_{\mathrm{appl}}=\phi_{\mathrm{m}}-\phi_{\mathrm{l}}\right)$ from Eq. 5 in conjunction with Eq. 8 , and then repeating these steps at successive points in time. The physical constants used to calculate the dimensionless parameters given in Fig. 3 are listed in Table II. The convergence of the series in Eq. 8 (and all other infinite series listed in this paper) was accelerated using Richardson extrapolation ${ }^{17}$ and a binomial averaging algorithm ${ }^{18}$ for $Y=1$ and $Y \neq 1$, respectively. Equation 5 was integrated numerically using Simpson's rule, and Newton's method was used to obtain $\eta(\tau)$ from Eq. 3 .

The three characteristics of the discharge curve observed in Fig. 2 are seen in Fig. 3, but some differences between the experimental and simulated curves do exist. For example, the initial voltage at $\mathbf{c} / 10$ lies on top of the one at $\mathbf{c} / 2$ in Fig. 2, but the corresponding simulated curve lies closer to the $\mathbf{c} / 20$ discharge. Also, the middle plateau and utilization changes more as the rate is increased from $\mathbf{c} / 20$ to $\mathbf{c} / 10$ in Fig. 2 than it does in Fig. 3. Even with these differences though, the model does reveal some important insight regarding the effect proton diffusion, electron conductivity, and charge-transfer resistance has on the nickel hydroxide discharge curves.

It is unclear from Fig. 3 what the relative contributions of mass-transfer, ohmic, and charge-transfer resistances are to the observed polarization losses. Figures 4-9 were generated in order to isolate the effects the dimensionless parameters listed in Table I have on the discharge curves. In Fig. 4, changes in the discharge curves as $I_{0}$ is decreased from $\infty$ to 0.3 are due solely to increases in charge-transfer resistance relative to discharge time. Mass-transfer and ohmic resistances were eliminated by replacing $C(1, \tau)(1-$ $\left.\theta^{\circ}\right)$ with $(1-\theta)$ in Eq. 3 and equating $\left(\phi_{s}-\phi_{1}\right)$ to $V_{\text {appl }}$ which

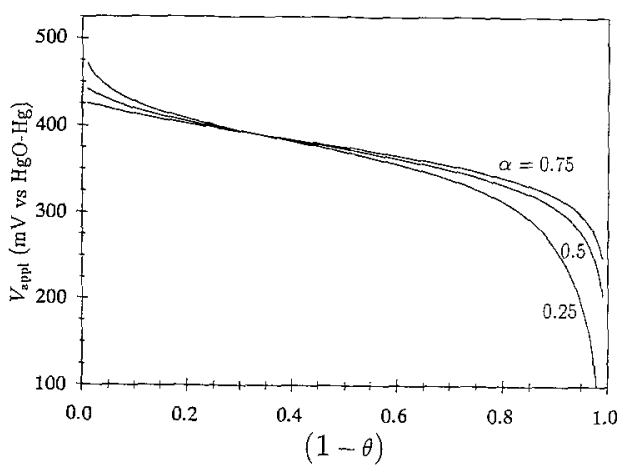

Fig. 5. The effect of the cathodic transfer coefficient on nickel hydroxide discharge curves. The discharge curves are only affected by $\alpha$ when charge-transfer resistance is appreciable. The applied voltages are plotted as a function of the average degree-of-discharge. $I_{0}=3 ; D=\sigma^{*}=\infty ; \theta^{\circ}=0.99 ; T=293$.

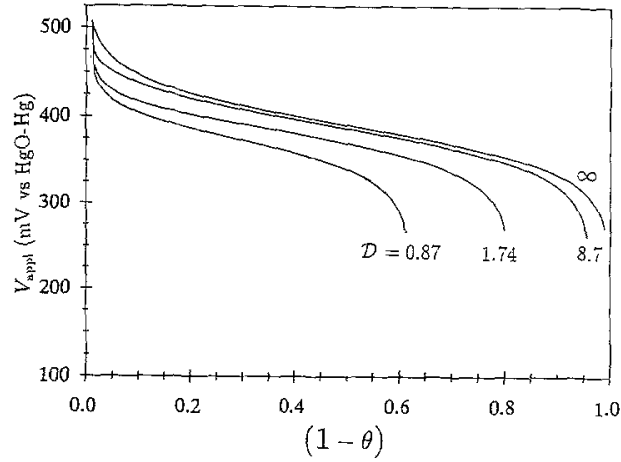

Fig. 6. The effect of proton mass-transfer resistance on nickel hydroxide discharge curves. As $\mathscr{D}$ increases, mass-transfer resistance decreases relative to discharge time. The applied voltages are plotted as a function of the average degree-of-discharge. $I_{0}=\sigma^{*}=\infty ; \alpha=0.5$; $\theta^{\circ}=0.99 ; T=293$.

is equivalent to setting $\mathscr{D}$ and $\sigma^{*}$ to infinity, respectively. The top curve in Fig. 4 is for $I_{o}=\infty$. This case was obtained by setting $I(\tau) / I_{o}=0$ in Eq. 3 which means that the applied voltage is the equilibrium potential.

Negligible change is observed as $I_{0}$ is decreased from $\infty$ to 30 . The $I_{0}=30$ case corresponds to the $\mathrm{c} / 20$ curve shown in Fig. 3 in the absence of ohmic and mass-transfer resistance. The fact that the $I_{0}=30$ curve shows very little change from the $I_{0}=\infty$ case indicates that none of the polarization losses observed in Fig. 3 at c/20 discharge is due to charge-transfer resistance. As $I_{0}$ decreases from 30 to 0.3 , charge-transfer resistance becomes increasingly important. The most significant effect of decreasing $I_{0}$ is the decrease in the voltage plateau. The $I_{0}=3$ curve corresponds to the $\mathbf{c} / 2$ curve in Fig. 3 in the absence of ohmic and mass-transfer resistance. Comparing these two curves reveals that charge-transfer resistance contributes approximately $15 \mathrm{mV}$ of polarization to the $\mathbf{c} / 2$ curve.

The sensitivity of charge-transfer resistance to discharge rate can be increased by decreasing the exchange current density an order-of-magnitude. That would result in the $I_{0}=3$ and 0.3 curves corresponding to $\mathrm{c} / 20$ and $\mathrm{c} / 2$ discharges, respectively (holding all other variables constant). The former discharge rate would have $15 \mathrm{mV}$ of polarization due to charge-transfer resistance, and the latter $100 \mathrm{mV}$. The strong dependence of charge-transfer resistance on discharge rate comes at the expense of a lack of curvature during the early portion of discharge. In addition, increases in charge-transfer resistance do not affect utilization as evident by the near $100 \%$ utilization at $I_{0}=$ 0.3 . Complete utilization regardless of discharge rate is the same result obtained from models that incorporate all resistances at the film/electrolyte interface into one pseudocharge-transfer resistance. ${ }^{1-5}$ 


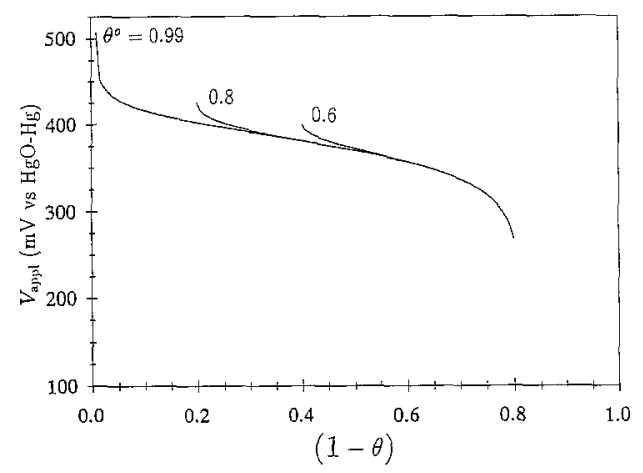

Fig. 8. The effect of the initial state-of-charge on nickel hydroxide discharge curves. The discharge curves are only affected by $\theta^{\circ}$ when mass-transfer resistance is appreciable. The applied voltages are plotted as a function of the average degree-of-discharge. $\mathscr{D}=1.74$; $\mathrm{I}_{0}=\sigma^{*}=\infty ; \alpha=0.5 ; T=293$.

Figure 5 shows the effect the cathodic transfer coefficient has on the nickel hydroxide discharge curve for $I_{0}=3$. At $I_{0}=30$, the discharge curves as $\alpha$ varies from 0 to 1 would be visually indistinguishable if plotted on Fig. 4. Only when charge-transfer resistance is significant does $\alpha$ affect the discharge curves. Since $\alpha$ only affects the discharge curves through the kinetic expression (Eq. 3), $\alpha$ was varied while $\mathscr{D}$ and $\sigma^{*}$ were set to infinity. The effect $\alpha$ has on the discharge curves is to cause more of an S shape as $\alpha$ is decreased from 0.75 to 0.25 . When $(1-\theta)$ is small, $\eta$ obtained from Eq. 3 is positive and decreasing $\alpha$ causes $\eta$ to become more positive which translates into a higher applied voltage. As $(1-\theta)$ is increased beyond $0.30, \eta$ is negative and decreasing $\alpha$ causes $\eta$ to become more negative which translate into a lower applied voltage. The sensitivity of the discharge curve on $\alpha$ will increase as $I_{0}$ decreases, and the effect described above will become more pronounced. Some of the $S$ shape lost in the discharge curve as $I_{\mathrm{o}}$ is decreased can be restored if $\alpha$ is decreased. Utilization though is not affected by $\alpha$ which further suggests that charge-transfer resistance is a minor contributor to determining the discharge characteristics of nickel hydroxide.

The polarization losses seen in Fig. 6 are due solely to the mass-transfer resistance of protons through the active nickel hydroxide film. The three characteristics seen in Fig. 3 are observed in Fig. 6. Diffusion limitations can account for potential decay during the early portions of discharge, and a voltage plateau and material utilization which are functions of discharge rate. The degree of this discharge-rate dependency, however, is a function of the diffusion coefficient and the film thickness. The $\mathbf{c} / 2$ and c/10 curves in Fig. 3 correspond to the $\mathscr{D}=1.74$ and 8.7 curves in Fig. 6, respectively, in the absence of chargetransfer and ohmic resistance. If the diffusion coefficient listed in Table $\Pi$ is increased an order-of-magnitude (or 1 is

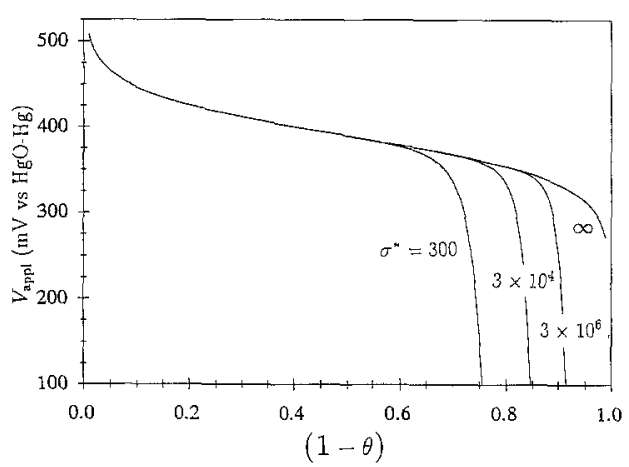

Fig. 9. The effect of ohmic resistance on nickel hydroxide discharge curves. As $\sigma^{*}$ increases, the ohmic resistance decreases relative to dischorge time. The applied voliages are plotted as a function of the average degree-of-discharge. $I_{0}=\mathscr{D}=\infty ; \alpha=0.5 ; \theta^{\circ}=0.99 ; T=298$. decreased by a factor of 3.16 ), the $\mathbf{c} / 2$ and $\mathbf{c} / 10$ case would correspond to $\mathscr{D}=17.4$ and 87 , respectively. From Fig. 6 , a D) greater than 8.7 is in a region where mass-transfer resistance becomes insignificant, and utilization approaches $100 \%$.

The reason 9 can have such a strong effect on the discharge curve is seen by examining the proton concentration profile in the film. Figure 7 shows the variation in the local degree-of-discharge at various values of $\mathscr{D}$ calculated using Eq. 8. The profiles correspond to the four curves shown in Fig. 6 at $(1-\theta)=0.5$. The point in the discharge at which the profiles are shown was chosen to allow significant changes in proton concentration to occur, but before discharge at $\mathscr{D}=1.74$ was terminated. At $\mathscr{D}=8.7$, the degreeof-discharge at the surface is nearly uniform and equal to the average value of 0.5 . Further increases in $D$ do not affect the surface concentration of protons since diffusion into the bulk of the solid is relatively fast and the polarization losses due to mass-transfer resistance is small. As masstransfer resistance increases, the degree-of-discharge at the surface is much greater than the average value of 0.5 , and a larger overpotential is required to maintain a constant-current discharge. A degree-of-discharge at the surface equal to one would require an infinity negative overpotential. Therefore, a $\mathscr{D}$ much smaller that 0.87 would not even reach $50 \%$ utilization. Figures 6 and 7 indicate that polarization losses due to the resistance of proton diffusion away from the film/electrolyte interface contribute significantly to the polarization losses observed in Fig. 3.

Figure 8 shows the effect the initial state-of-charge has on the discharge curves when mass-transfer resistance of protons is appreciable, but ohmic and charge-transfer resistance is zero. The reason this case is shown is that varying $\theta^{\circ}$ on Fig. 4 and 9 would not affect the potential at a given $(1-\theta)$. Hysteresis is only possible when mass-transfer limitations are significant. A quickly decaying voltage is observed in Fig. 8 regardless of the point at which discharge begins due to the sharp concentration gradient established after the switch is closed. As the discharge progresses, the gradients smooth out and the effect $\theta^{\circ}$ has on the later stages of discharge decreases. Knowing the initial state-of-charge is important since the utilization one determines from the discharge curve is dependent on the value assumed for $\theta^{\circ}$. It was assumed in plotting Fig. 2 that the electrode was initially fully charged. If in reality $\theta^{\circ} \sim 0.85$, the $\mathbf{c} / 20$ discharge curve would have approached $1.00 \%$ utilization. An incorrect assumption that the electrode was fully charged could also explain why the initial voltage at c/10 lies on top of the one at c/2 in Fig. 2, and why the middle plateau and utilization changes more as the rate is increased from $\mathrm{c} / 20$ to $\mathrm{c} / 10$ in the experimental curves than they do in the simulated ones. If, for instance, the c/ 10 discharge had an initial state-of-charge of 0.95 instead of 1.0, the experimental curves in Fig. 2 would more closely resemble the simulated curves in Fig. 3.

Figure 9 shows the polarization losses due to ohmic resistance. The only effect a decrease in $\sigma^{*}$ has on the discharge curves is a decrease in utilization. Neither potential decay during the early portions of discharge nor the voltage plateau are affected by the four order-of-magnitude change in $\sigma^{*}$ shown in Fig. 9. These results indicate that the conductivity during a major portion of discharge contributes very little to the overall polarization losses seen in Fig. 3. The conductivity at the end of discharge does, however, cause significant polarization losses. A point is reached where the conductivity of the hydroxide layer is so small that the applied voltage must be removed several hundred millivolts from equilibrium in order to sustain the desired discharge current. Knowing the reduced-phase conductivity is important in order to correctly predict utilization.

The loss in electrode utilization resulting from low reduced-phase conductivity is not as sensitive to discharge rate as can be the case with mass-transfer resistance. The $\sigma^{*}=3 \times 10^{4}$ curve in Fig. 9 has the identical ohmic resistance as does the c/2 discharge in Fig. 3 . Ohmic resistance alone allows approximately $85 \%$ of the electrode to be uti- 
lized. Decreasing the discharge rate to $\mathrm{c} / 200$ makes $\sigma^{*}=$ $3 \times 10^{6}$ which only increases utilization to slightly over $90 \%$. Increasing the discharge rate to $50 \mathrm{c}\left(\sigma^{*}=300\right)$ only decreases utilization to $75 \%$. In contrast, Fig. 6 shows that increasing $\mathscr{D}$ from 0.87 to 1.74 to 8.7 (equivalent to decreasing discharge rate from $\mathbf{c}$ to $\mathbf{c} / 2$ to $\mathbf{c} / 10$ if the physical constants in Table II are used), increases utilization from 60 to 80 to $95 \%$, respectively.

Unknown current density.-The validity of the integral approximation in Eq. 9 was tested by generating discharge curves using Eq. 13 in place of Eq. 8 with $I(z)=-1$. The potentials at a given $(1-\theta)$ obtained using Eq. 13 were within $0.1 \mathrm{mV}$ of those obtained using Eq. 8. The resulting discharge curves would be visually indistinguishable if plotted in Fig. 3-6, 8, and 9. Therefore, incorporating Eq. 13 into a one-dimensional macrohomogeneous model should provide a computationally efficient means of accounting for proton diffusion and variable electronic conductivity without sacrificing accuracy.

\section{Conclusions}

Constant-current discharge curves for the nickel hydroxide electrode are simulated assuming resistances due to diffusion of protons and conduction of electrons through the active nickel hydroxide film as well as charge-transfer resistance at the film/electrolyte interface contribute to the polarization losses of the electrode. The model predicts three characteristics of the discharge curve which are observed experimentally: (i) a quickly decaying voltage during the early portion of discharge; (ii) a voltage plateau during the middle portion of discharge which decreases as the discharge rate increases; (iii) material utilization which decreases as discharge rate increases. The results suggest that polarization losses due to diffusional limitations of protons is a critical factor in determining the characteristics of the discharge curve. Ohmic resistance has a significant effect on the discharge curves at the end of discharge, and charge-transfer resistance is a minor contributor to the polarization losses.

The simulations also indicate which physical constants are needed to accurately predict nickel hydroxide discharge curves. The critical constants are the diffusion coefficient of protons, the thickness of the hydroxide film, the initial state-of-charge, and the electronic conductivity as a function of state-of-charge towards the end of discharge. Physical constants which were shown to be less significant for accurately predicting the discharge behavior of the nickel hydroxide are the film conductivity at the beginning of discharge, and the exchange current density, and cathodic transfer coefficient for the reaction.

In order to predict the discharge behavior of a nickel battery, it will be important to know how the resistances resulting from the hydroxide film interact with the resistances caused by the entire cell. The time-dependent, onedimensional diffusion equation has been solved analytically which should provide a computationally efficient means of accounting for proton diffusion and variable electronic conductivity in a macrohomogeneous battery model without sacrificing accuracy.

Manuscript submitted July 26, 1993; revised manuscript received Oct. $21,1993$.

The University of South Carolina assisted in meeting the publication costs of this article.

\section{LIST OF SYMBOLS}

c capacity of electrode, $\mathrm{C}$

$c_{\mathrm{H}^{+}}$proton concentration, $\mathrm{mol} / \mathrm{cm}^{3}$

$c_{\mathrm{H}^{+}}^{\circ}$ initial proton concentration, $\mathrm{mol} / \mathrm{cm}^{3}$

$c_{\mathrm{Ni}}$ concentration of nickel sites, $\mathrm{mol} / \mathrm{cm}^{3}$
C dimensionless proton concentration, $c_{\mathrm{H}^{+}} / c_{\mathrm{H}^{+}}^{\circ}$

$D_{\mathrm{H}^{+}}$diffusion coefficient for protons, $\mathrm{cm}^{2} / \mathrm{s}$

D $3600 n^{\prime} D_{\mathrm{H}^{+}} / l^{2}$

f F/RT, $\mathrm{V}^{-1}$

F Faraday's constant, 96,485 C/equiv

$i$ current density, $\mathrm{A} / \mathrm{cm}^{2}$

$i_{0, \text { ref }}$ exchange current density at reference state, $\mathrm{A} / \mathrm{cm}^{2}$

$I$ dimensionless current density, $3600 n^{\prime} i / F c_{\mathrm{Ni}} l$

$I_{\mathrm{o}} \quad 3600 n^{\prime} i_{\mathrm{o} \text {,ret }} / F^{\prime} c_{\mathrm{Ni}} l$

$l$ thickness of the nickel hydroxide layer, $\mathrm{cm}$

$n$ hours required to discharge the nominal capacity, $h$

$n^{\prime} \quad$ hours required to discharge the maximum capacity, $h$

$R \quad$ gas constant, $8.314 \mathrm{~J} / \mathrm{mol} \cdot \mathrm{K}$

$t$ time, $s$

$T$ temperature, $\mathrm{K}$

$U_{\text {ref }}$ equilibrium potential at reference state, $\mathrm{V}$

$V_{\text {appl }}$ applied voltage, $\left(\phi_{\mathrm{m}}-\phi_{1}\right), \mathrm{V}$

$x_{\mathrm{j}} \quad$ mole fraction of species $\mathrm{j}$ in hydroxide film, $\mathrm{j}=\mathrm{NiOOH}$ and $\mathrm{Ni}(\mathrm{OH})_{2}$

$y \quad$ distance into hydroxide film, $\mathrm{cm}$

$Y \quad$ dimensionless distance, $y / l$

Greek

$\alpha \quad$ cathodic transfer coefficient

$\eta \quad$ reference overpotential, $\left(\phi_{\mathrm{s}}-\phi_{1}-U_{\text {ref }}\right), \mathrm{V}$

$\theta \quad$ state-of-charge, $\left(\theta^{\circ}-t / 3600 n^{\prime}\right)$

$\theta^{\circ} \quad$ initial state-of-charge

$\sigma^{*} \quad 3600 n^{\prime} \sigma^{\circ} / f F c_{\mathrm{Ni}} l^{2}$

$\sigma^{\circ} \quad$ initial conductivity, $(\Omega \cdot \mathrm{cm})^{-1}$

$\phi$ potential, $\mathrm{V}$

$\tau$ dimensionless time, $t D_{\mathrm{H}^{+}} / l^{2}$

Subscripts

1 electrolyte phase

$\mathrm{m}$ matrix phase

s hydroxide film at $Y=1$

\section{REFERENCES}

1. K. W. Choi and N. P. Yao, in Proceedings of the Symposium on Battery Design and Optimization, S. Gross, Editor, PV 79-1, p. 50, The Electrochemical Society Proceedings Series, Princeton, NJ (1979).

2. K. Micka, and I. Roušar, Electrochim. Acta, 25, 1085 (1980).

3. K. Micka and I. Roušar, ibid., 27, 765 (1982).

4. D. Fan and R. E. White, This Journal, 138, 17 (1991).

5. D. Fan and R. E. White, ibid., 138, 2952 (1991).

6. M. Sinha, Ph.D. Thesis, University of California, Los Angeles (1982).

7. G. W. D. Briggs, and P. R. Snodin, Electrochim. Acta, 27, 565 (1982).

8. A. H. Zimmerman and P. K. Effa, This Journal, 131, 709 (1984).

9. C. Zhang and S. Park, ibid., 134, 2966 (1987).

10. R. W. Crocker and R. H. Muller, Report, LBL-32417 (1992).

11. J. Bouet, F. Richard, and P. Blanchard, in Proceedings of the Symposium on Nickel Hydroxide Electrodes, D. A. Corrigan and A. H. Zimmerman, PV90-4, p. 260, The Electrochemical Society Proceedings Series, Pennington, NJ (1990).

12. Z. Mao, P. De Vidts, R. E. White, and J. Newman, This Journal, 141, 54 (1994).

13. S. U. Falk and A. J. Salkine, Alkaline Storage Batteries, p. 533, John Wiley \& Sons, Inc., New York (1969).

14. R. Barnard, G. T. Crickmore, J. A. Lee, and F. L. Tye, J. Appl. Electrochem., 10, 61 (1980).

15. P. Delahay, J. Am. Chem. Soc., 75, 1190 (1953).

16. P. J. Timmerman, S. Di Stefano, P. R. Glück, and D. E. Perrone, in Proceedings of IECEC, Boston, MA, Vol. 3, p. 358 (Aug. 1991).

17. C. M. Bender and S. A. Orszad, Advanced Mathematical Methods for Scientists and Engineers, McGraw Hill, Inc., New York (1978).

18. G. Dahlquist, and Å. Björk, Numerical Methods, Prentice Hall, Inc., Englewood Cliffs, NJ (1974); Translated by $\mathrm{N}$. Anderson. 